\title{
An Online Implementable Differential Evolution Tuned Optimal Guidance Law
}

\author{
Raghunathan Thangavelu \\ Indian Institute of Science \\ Ph.D. student \\ Aerospace Engineering Department \\ Bangalore - 560012, India \\ 91-9449987234 \\ raghu_t@aero.iisc.ernet.in
}

\author{
S. Pradeep \\ Indian Institute of Science \\ Associate Professor \\ Aerospace Engineering Department \\ Bangalore - 560012, India \\ 91-80-22932480 \\ spradeep@aero.iisc.ernet.in
}

\begin{abstract}
This paper proposes a novel application of differential evolution to solve a difficult dynamic optimisation or optimal control problem. The miss distance in a missile-target engagement is minimised using differential evolution. The difficulty of solving it by existing conventional techniques in optimal control theory is caused by the nonlinearity of the dynamic constraint equation, inequality constraint on the control input and inequality constraint on another parameter that enters problem indirectly.

The optimal control problem of finding the minimum miss distance has an analytical solution subject to several simplifying assumptions. In the approach proposed in this paper, the initial population is generated around the seed value given by this analytical solution. Thereafter, the algorithm progresses to an acceptable final solution within a few generations, satisfying the constraints at every iteration. Since this solution or the control input has to be obtained in real time to be of any use in practice, the feasibility of online implementation is also illustrated.
\end{abstract}

\section{Categories and Subject Descriptors}

I.2.8 [Problem Solving, Control Methods, and Search]: Heuristic methods

\section{General Terms}

Algorithms

\section{Keywords}

Missile guidance, optimal guidance law, evolutionary algorithm, differential evolution, optimal control, online implementation

\section{INTRODUCTION}

Evolutionary algorithms (EAs) have been eminently successful in solving static optimisation problems where traditional hill

Permission to make digital or hard copies of all or part of this work for personal or classroom use is granted without fee provided that copies are not made or distributed for profit or commercial advantage and that copies bear this notice and the full citation on the first page. To copy otherwise, or republish, to post on servers or to redistribute to lists, requires prior specific permission and/or a fee.

GECCO'07, July 7-11, 2007, London, England, United Kingdom.

Copyright 2007 ACM 978-1-59593-697-4/07/0007...\$5.00. climbing methods have failed. However, their performance as general purpose optimisers have been sub-par for problems solvable by traditional methods, since they fail to exploit problem specific features like gradient information that can lead to quick convergence to the optimum solution. Not surprisingly, most applications of EAs have been for static optimisation problems.

For dynamic optimisation or optimal control applications, EAs could be employed for finding solutions online, under any of the following circumstances [1]: (i) the plant or system model is available, and dynamics is slow enough to allow the computation time needed by the EA to arrive at the solution (ii) a 'good enough' solution, not necessarily the best, is all that is needed for the application (iii) massively parallel implementation is possible, so that the best solution is obtained in a small interval of time.

However, the missile guidance problem falls in none of the above three categories. The dynamics is very fast, and the best solution (least miss distance) is the one that is acceptable. Though computational capacities of computers have been increasing rapidly, the onboard computing power on the missile is still not large enough to allow massively parallel implementation. To overcome the above difficulties, this paper proposes a two loop hybrid control.

This paper is organised as follows: section 2 gives a brief introduction to the guidance problem from a perspective necessary to develop the paper. The problem formulation and solution methodology is developed in section 3. It is followed by implementation and results in section 4 and the concluding remarks in section 5 .

\section{MISSILE GUIDANCE AS AN OPTIMAL CONTROL PROBLEM}

\subsection{The simplified linear model}

The linear state model of the missile target engagement (Figure 1) is given by [2]

$$
\left[\begin{array}{c}
\dot{y} \\
\ddot{y} \\
\dot{n}_{T} \\
\dot{n}_{L}
\end{array}\right]=\left[\begin{array}{cccc}
0 & 1 & 0 & 0 \\
0 & 0 & 1 & 0 \\
0 & 0 & 0 & 0 \\
0 & 0 & 0 & -1 / T
\end{array}\right]\left[\begin{array}{c}
y \\
\dot{y} \\
n_{T} \\
n_{L}
\end{array}\right]+\left[\begin{array}{c}
0 \\
-1 \\
0 \\
1 / T
\end{array}\right] n_{c}
$$


In this linear time-varying system, missile acceleration $n_{c}$ is subtracted from target acceleration $n_{T}$ to the form the relative acceleration $\ddot{y}$. A double integration yields the relative vertical position $y$, which at the end of the engagement, $t=t_{F}$, is the miss distance $y\left(t_{F}\right)$. By assuming that the closing velocity $V_{c}$ is constant, the relative range at any time $t$ during the flight time $t_{F}$ is given by

$$
R_{T M}=V_{c} t_{g o}, \quad \text { where } \quad t_{g o}=t_{F}-t
$$

Linear quadratic optimal control theory can be applied to find the optimal control that minimises the performance index

$$
J=\int_{0}^{t_{F}} n_{c}^{2} d t
$$

subject to the state equation (1), and

$$
y\left(t_{F}\right)=0
$$

where $y\left(t_{F}\right)$ is the miss distance.

The control input that achieves this is given by

$$
n_{c}=\frac{N^{\prime}}{t_{g o}^{2}}\left[y+\dot{y} t_{g o}+0.5 n_{T} t_{g o}^{2}-n_{L} T^{2}\left(e^{-x}-1+x\right)\right]
$$

where $\quad x=t_{g o} / T=\left(t_{F}-t\right) / T$

and

$$
N^{\prime}=\frac{6 x^{2}\left(e^{-x}-1+x\right)}{2 x^{3}+3+6 x-6 x^{2}-12 x e^{-x}-3 e^{-2 x}}
$$

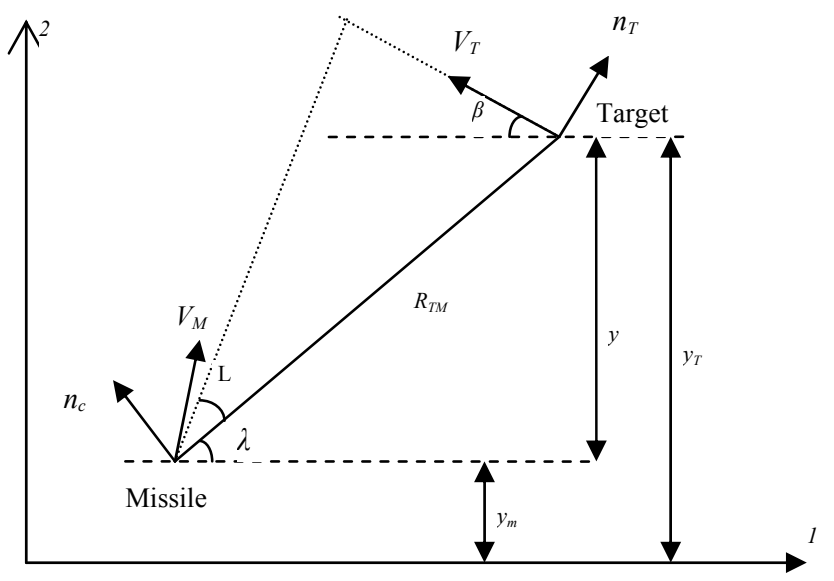

Figure 1. Two-dimensional missile-target engagement model

(3) is the well known Optimal Guidance Law (OGL). The OGL works on the basis of the cancellation of guidance system dynamics which is given by $\frac{n_{L}}{n_{c}}=\frac{1}{1+s T}$.

The OGL assumes that unconstrained control input is available; in other words, commanded acceleration $n_{c}$ saturation does not occur. If the input is not unconstrained, it results in a non-zero miss distance. Equation (2b) would no longer be satisfied.

In order to obtain the above analytical solution, certain simplifying assumptions have been imposed: the state equation constraint describing the kinematics of the missile-target is linear (given by (1) above), the control (3a) is unconstrained, and $N^{\prime}$ too is unconstrained (3c) [2]. However, in practice, these assumptions prove to be too strong to reflect the reality. The kinematics involved is known to be highly nonlinear [3], and the control available is limited by the acceleration saturation that every physical system like a missile is subject to. $N^{\prime}$ too faces a lower and upper bound [2].

\subsection{The nonlinear engagement model}

A more exact, nonlinear model of the missile-target engagement is given by

$$
\left[\begin{array}{c}
\dot{\beta} \\
\dot{R}_{T 1} \\
\dot{R}_{T 2} \\
\dot{V}_{M 1} \\
\dot{V}_{M 2} \\
\dot{R}_{M 1} \\
\dot{R}_{M 2} \\
\dot{n}_{L}
\end{array}\right]=\left[\begin{array}{c}
n_{T} / V_{T} \\
-V_{T} \cos \beta \\
V_{T} \sin \beta \\
-n_{L} \sin \lambda \\
n_{L} \cos \lambda \\
V_{M 1} \\
V_{M 2} \\
\left(-n_{L}+n_{c}\right) / T
\end{array}\right]
$$

where $\quad \lambda=\tan ^{-1}\left(R_{T M 2} / R_{T M 1}\right), \quad n_{L}$ is the actual acceleration that results when acceleration $n_{c}$ is commanded, and $T$ is the time-constant of the missile guidance system. The subscript ' 1 ' denotes the component along axis 1 and the subscript ' 2 ' the component along axis 2 . For instance, $\dot{R}_{T 1}$ is the component of $\dot{R}_{T}$ along axis 1 , and $\dot{R}_{T 2}$ the component of $\dot{R}_{T}$ that along axis 2. $R_{M}=\left(R_{M 1}, R_{M 2}\right)$ and $R_{T}=\left(R_{T 1}, R_{T 2}\right)$ are the positions of the missile and the target respectively. $R_{T M}=\left(R_{T M 1}, R_{T M 2}\right)$ is the relative separation of the missile and the target, where $R_{T M 1}=R_{T I}$ - $R_{M 1}$, and $R_{T M 2}=R_{T 2}-R_{M 2}$. The acceleration $n_{c}$ is the control input to the plant (4).

\section{PROBLEM FORMULATION 3.1 The Mayer type optimal control formulation}

Application of the OGL to the linear model (1) to the numerical example in section 4 shows that the OGL produces very low miss distances as the missile-target separation is varied. However, application of the OGL for the more realistic nonlinear model (4) shows large miss distances as the missile-target separation and consequently the flight time increase (Figure 2). The reasons for this are explained in [4], [5].

Reduction of the miss distance is possible by solving the more exact, nonlinear Mayer form optimal control problem [6] described below:

$$
\text { Minimise } J\left(n_{c}(t)\right)=X^{T}\left(t_{F}\right) \boldsymbol{S} X\left(t_{F}\right)
$$


subject to the nonlinear kinematics (4), which is of the form

$$
\begin{aligned}
& \dot{X}=f\left(X, n_{c}\right) \\
& \left|n_{c}\right| \leq n_{c, \max } \\
& X\left(t_{0}\right)=X_{0}, X\left(t_{F}\right) \text { free, and } t_{F} \text { free }
\end{aligned}
$$

However, the solution (5) is far more difficult than the solution of (2) [5], [7]

The present paper proposes a simpler, but not as widely applicable method as the one in [8]: make use of the closed form solution (3) that is available for the linearised model. This can serve as a good starting point for generating trial solutions around it, and the best solution can be found thereafter by a stochastic search technique like differential evolution (DE). The approach herein also makes online implementation feasible.

\subsection{The Proposed Approach}

Next, three heuristic propositions that are central to the proposed approach are made in this paper.

Proposition 1: A common, but approximate way of estimating the flight time $\hat{t}_{F}$ is

$$
\hat{t}_{F}=R_{T M}(0) / V_{c}(0)
$$

where ' $\wedge$ ' is used to indicate that an estimated value is being referred to. Since (6) is only approximate, treat $t_{F}$ as variable within a certain upper and lower bound:

$$
0.8 \hat{t}_{F}<t_{F}<1.2 \hat{t}_{F}
$$

Proposition 2: Even if accurate $t_{F}$ is available, still treat $t_{F}$ as variable, as per proposition 1 . This is because the OGL can give a large miss distance when applied to the nonlinear plant even with accurate $t_{F}$.

Proposition 3: The guidance system dynamics has a number of time constants, either widely differing from each other in magnitude, or closely spaced. If the time constants are of widely differing magnitudes, the slowest time constant $\hat{T}$ will usually dictate the system performance. If they are closely spaced, they can be conveniently represented as a binomial representation [1]:

$$
\frac{n_{L}}{n_{c}}=\frac{1}{\left(1+\frac{s T}{n}\right)^{n}}
$$

Expanding the denominator as a binomial series,

$$
\left(1+\frac{s T}{n}\right)^{n}=1+s T+\ldots \approx 1+s \hat{T}
$$

Regardless of whether the time constants are of widely differing magnitudes, or closely spaced, the missile system dynamics can always be represented by a single, approximate time constant $\hat{T}$.

$$
\begin{aligned}
& \text { Hence treat } T \text { as variable within the bounds } \\
& \frac{T}{\hat{T}} \in[0.8,1.2]
\end{aligned}
$$

\subsection{Problem formulation}

With $t_{F}$ and $T$ as the variables, bounded by

$$
\begin{aligned}
& \frac{t_{F}}{\hat{t}_{F}} \in[0.8,1.2] \\
& \frac{T}{\hat{T}} \in[0.8,1.2]
\end{aligned}
$$

solve the following optimisation problem:

$$
\begin{aligned}
& \text { Minimise } J=R_{T M}\left(t_{F}\right) \\
& \text { subject to } \\
& \text { the nonlinear kinematics (3) } \\
& 0 \leq n_{c} \leq n_{c, \max } \\
& -5 \leq N^{\prime} \leq 5
\end{aligned}
$$

As compared to the optimal control problem formulation (5), the approach proposed in the present paper is much simpler: start with a set of randomly generated feasible solutions seeded with the values of the input variables $\hat{t}_{F}$ and $\hat{T}$ to the OGL, and use an intelligent algorithm to improve upon these feasible solutions and arrive at the best solution iteratively.

The missile-target kinematics (7d) and the bounds constraints (7e) and (7f) can be treated as a black box, with its input as $t_{F}$ and $T$ and its output as the miss distance $R_{T M}\left(t_{F}\right)$. The control input $n_{c}=n_{c}\left(t_{F}, T, y, \dot{y}\right)$ varies as the quantities $t_{F}$ and $T$ are varied, in turn varying the output $R_{T M}\left(t_{F}\right)$ of the black box. The control input is fixed at the bound, if it exceeds any bound in its time history; similarly $N^{\prime}$ is fixed at the bound if it exceeds any bound. The minimum $R_{T M}\left(t_{F}\right)$ can be found by an exhaustive search by varying the input variables over their whole range. However, an intelligent search technique employing directed random search like any EA can produce a quicker solution. The nominal values

of $\hat{t}_{F}$ given by (7) and $T$ (specified in the problem data) are used to seed the initial population.

\section{IMPLEMENTATION AND RESULTS}

The proposed method is implemented in the following numerical example [2], [5].

$$
\begin{aligned}
& R_{M 1}=0, R_{M 2}=10,000 \mathrm{ft}, R_{T 1}=40,000 \mathrm{ft} \\
& R_{T 2}=10,000 \mathrm{ft}, V_{M}=3,000 \mathrm{ft} / \mathbf{s}, V_{T}=1,000 \mathrm{ft} / \mathrm{s} \\
& n_{T}=96.6 \mathrm{ft} / \mathbf{s}^{2}=3 \mathrm{~g}
\end{aligned}
$$

The heading error (HE) was assumed to be zero.

$n_{c, \max .}=3 n_{T}=9 \mathrm{~g}$.

The maximum value of $N^{\prime}$ was taken as 5, for the OGL. The approximate system time constant $T$ in (1) was taken to be $1 \mathrm{~s}$. 
The initial population comprises individuals that are the randomly generated pairs of $\hat{t}_{F}$ and $\hat{T}$ about the seed values of $T=1 \mathrm{~s}$, and $\hat{t}_{F}=R_{T M}(0) / V_{c}(0)=40,000 / 4,000=10 \mathrm{~s}$. The fitness of each trial solution or individual is evaluated by solving $(7 \mathrm{~d})$ with the OGL as the control input, and obtaining the miss distance as the output. The best solution is that pair of $t_{F}$ and $T$ which gives a miss distance less than the tolerance during the generations, or the least miss distance after the maximum number of generations allowable.

DE was chosen out of all EAs available, to speed up the execution, since the DE algorithm is one of the simplest of all EAs, and hence very suitable for quick execution. The population size was 12, and the stopping criterion was fixed as the earlier of the tolerance limit of 3 feet of miss distance, or a maximum of four generations. All these tuning parameters were arrived at empirically, to give the minimum run time.

Even with DE, the missile dynamics is too fast to allow the optimal solution to be determined online using a computationally intensive method like any EA. Hence a two-loop hybrid control is proposed herein (Figure 3). The upper loop comprising OGL would be in action till some intermediate time interval $T_{l}$ where $0<T_{I}<t_{F}$, during which the DE-OGL would arrive at the best solution. At $T_{I}$, the upper loop would stop functioning, and from $T_{1}$ onwards, the DE-OGL would be applied. For the example problem in this paper, our implementation of the DE-OGL needs a maximum of $T_{1}=2$ seconds to calculate the control needed. Clearly, DE-OGL can be applied to the plant only for flight times $t_{F} \geq 2 T_{1}$. However, for small $t_{F} \mathrm{~s}$, there is no need for DE-OGL, since the small angle approximation is still valid, and performance of the OGL itself is quite good. For the example problem in this paper, which started with head-on encounter initial conditions, OGL performs well up to almost 6 seconds of flight time (Figure 2).

The coding and simulation was done in Matlab ${ }^{\circledR}$ language. The fourth-order Runge-Kutta method was used to solve (4) with a step size of 0.01 second. The DE implemented used binomial crossover, with a crossover constant (CR) of 0.9 , and weighting factor $(\mathrm{F})$ of 0.8 , with parts of the code adapted from [9]. The implementation was done on a Pentium IV, $3 \mathrm{MHz}$ computer with 512 MB RAM and 4 GB virtual memory.

Figure 2 shows the miss distances obtained for various $t_{F}$ 's, for various initial separations of the missile and target. It can be seen that over the entire range of $t_{F}$ 's, the proposed DE-OGL performs as well as OGL, or even better, in terms of miss distance. Figures 4 and 5 give an indication of how this is achieved. The proposed method dictates higher values of $n_{c}$ and $N^{\prime}$ in the initial stage than the OGL. It is to be noted that the control input $n_{c}$ is partly singular (the initial part of the flight) and partly bang-bang (from about 6.92s, approx.). The improvement in solutions (miss distance) as the generations proceed is shown in Figure 6.

\section{CONCLUDING COMMENTS}

A new differential evolution tuned optimal guidance law that performs better than the conventional OGL has been presented.

A limitation of the proposed method is that the range of operating conditions has been only extended; it applicability for highly nonlinear operating conditions is still to be improved, unlike in [8], which applies to all initial conditions. However, the approach in [8] was an offline solution, while the method herein is onlineimplementable. The approach in [10] too applies to larger range of operating conditions, but it does not consider saturation of control, an important practical constraint. Moreover, the approach therein too was for a single set of initial conditions, and redoing the exercise was necessary for a different set of initial conditions, as the authors themselves point out.

Another point to be noted is that the method herein assumed constant target manoeuvre; its performance for random target manoeuvre is yet to be studied.

\section{REFERENCES}

[1] Linkens, D. A., and Nyongesa, H. O., "Genetic algorithms for fuzzy control part 2; online system development and application", IEE Proc. - Control Theory Appl., Vol. 142, No. 3, May 1995.

[2] Zarchan P., Tactical and Strategic Missile Guidance, Progress in Astronautics and Aeronautics, Vol. 199, Fourth Ed., AIAA, Reston, Virginia, 2002.

[3] Chen, B. S., Chen, Y. Y., and Lin, C. L., 'Nonlinear Fuzzy $\mathrm{H} \infty$ Guidance Law With Saturation of Actuators Against Maneuvering Targets', IEEE Transactions on Control Systems Technology, vol. 10, no. 6, Nov. 2002, pp. 769-779.

[4] J. Shinar, and T. Shima, 'Guidance Law Evaluation in Highly Nonlinear Scenarios - Comparison to Linear Analysis,' in AIAA Guidance, Navigation and Control Conference, 1999, Paper no. 99-4065, pp. 651-661.

[5] Thangavelu. R., and Pradeep, S., 'A Differential Evolution Tuned Optimal Guidance Law,' in Mediterannean Conference on Control and Automation, June 2007, Paper no. T13-002.

[6] D. S. Naidu, Optimal Control Systems, CRC Press LLC, Boca Raton, Florida, 2003

[7] John T. Betts, Practical Methods for Optimal Control Using Nonlinear Programming, Society for Industrial and Applied Mathematics, Philadelphia, 2001, pp. 84.

[8] H. Seywald, R. R. Kumar, and S. M. Deshpande, "Genetic Algorithm Approach for Optimal Control Problems with Linearly Appearing Controls," Journal of Guidance, Control, and Dynamics, Vol. 18, No. 1, Jan.-Feb. 1995, pp. 177-182.

[9] Differential Evolution home page. Available: http://www.icsi.berkeley.edu/ storn/code.html

[10] Y. C. Sim, S. B. Leng, and V. Subramaniam, “A combined genetic algorithms-shooting method approach to solving optimal control problems," International Journal of Systems Science, Vol 31, No. 1, 2000, pp. 83-89. 


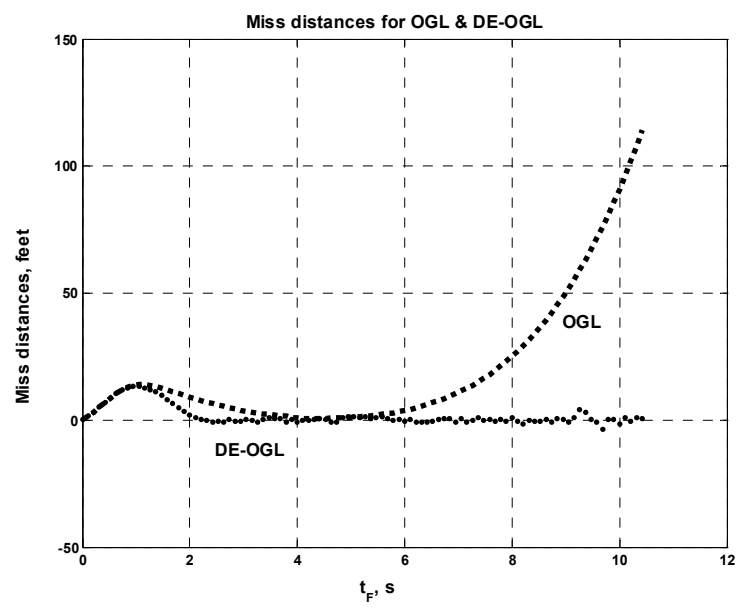

Figure 2. Miss distances of the OGL and DE-OGL

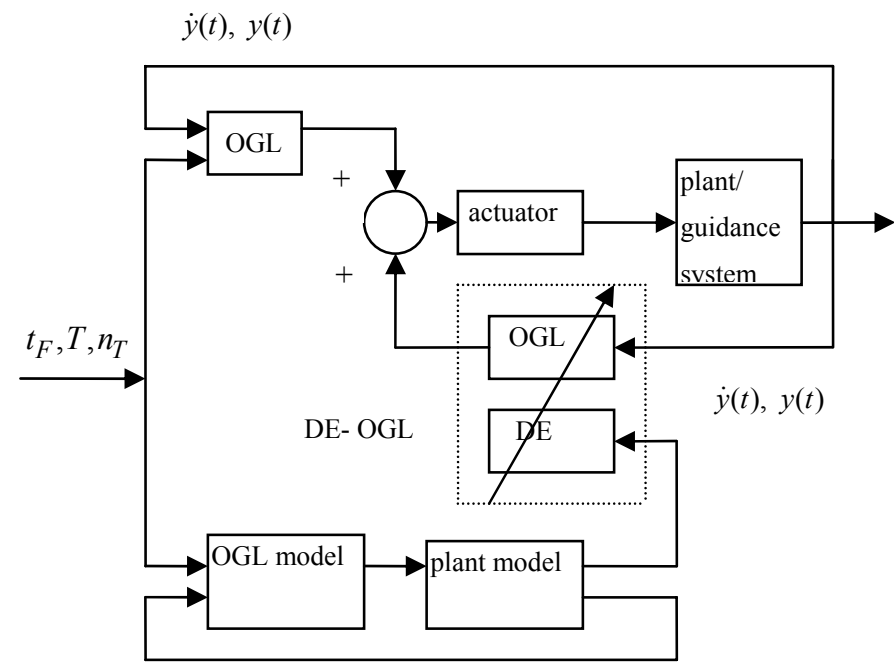

$\dot{y}(t), \quad y(t)$

Figure 3. Online implementation of DE-OGL

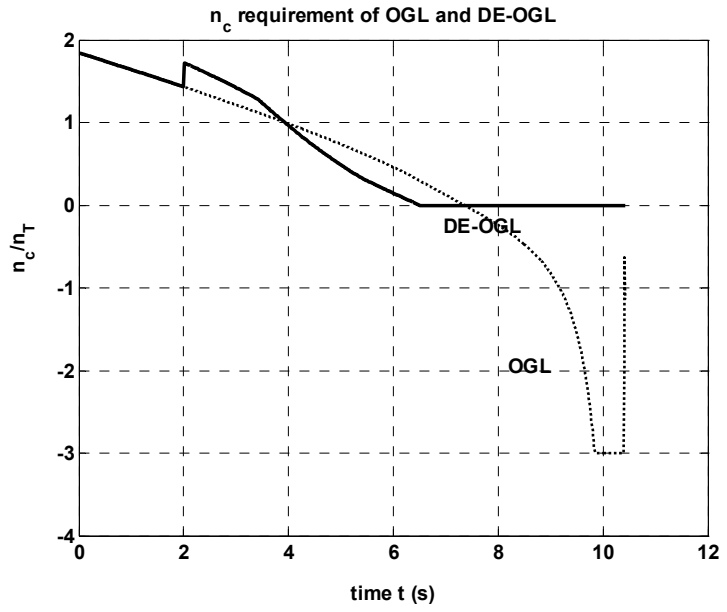

Figure 4. $n_{c}$ requirement of OGL and DE-OGL

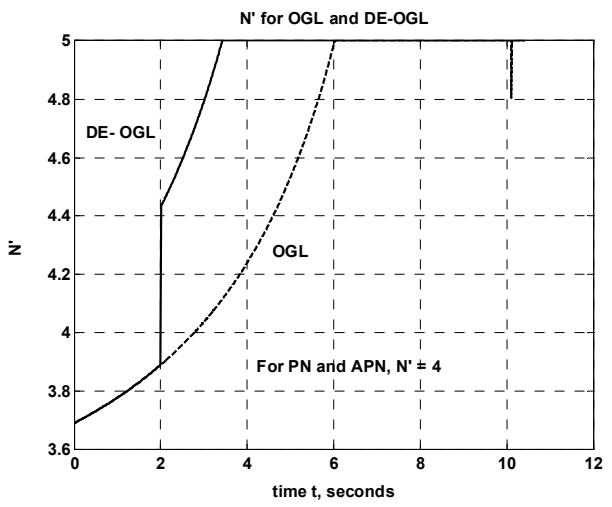

Figure 5. $N$ ' requirement of the OGL and DE-OGL

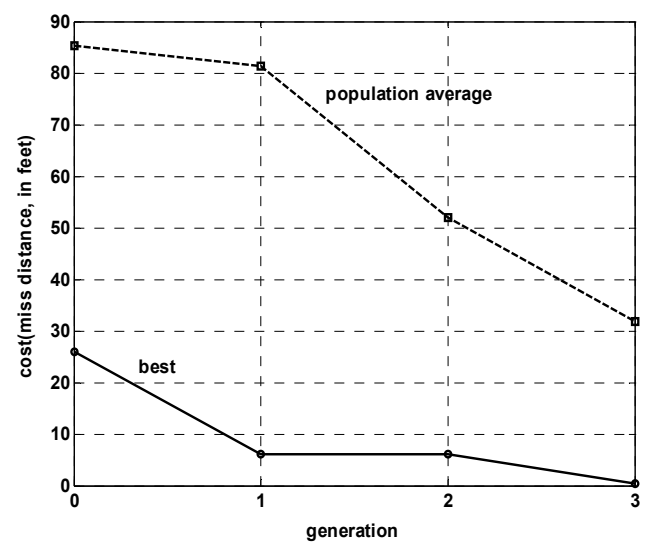

Figure 6. Improvement of the solution through the generations 